\title{
Lipocalin 2 contributes to brain iron dysregulation but does not affect cognition, plaque load, and glial activation in the J20 Alzheimer mouse model
}

Doortje W. Dekens ${ }^{1,2}$, Petrus J. W. Naudé1,2, Jan N. Keijser ${ }^{2}$, Ate S. Boerema ${ }^{2,3}$, Peter P. De Deyn ${ }^{1,4}$ and Ulrich L. M. Eisel ${ }^{2,5^{*}}$ (1)

\begin{abstract}
Background: Lipocalin 2 (LCn2) is an acute-phase protein implicated in multiple neurodegenerative conditions. Interestingly, both neuroprotective and neurodegenerative effects have been described for Lcn2. Increased Lcn2 levels were found in human post-mortem Alzheimer (AD) brain tissue, and in vitro studies indicated that Lcn2 aggravates amyloid- $\beta$-induced toxicity. However, the role of Lcn2 has not been studied in an in vivo AD model. Therefore, in the current study, the effects of Lcn2 were studied in the J20 mouse model of AD.

Methods: J20 mice and Lcn2-deficient J20 (J20xLcn2 KO) mice were compared at the behavioral and neuropathological level.

Results: J20xLcn2 KO and J20 mice presented equally strong AD-like behavioral changes, cognitive impairment, plaque load, and glial activation. Interestingly, hippocampal iron accumulation was significantly decreased in J20xLcn2 KO mice as compared to J20 mice.
\end{abstract}

Conclusions: Lcn2 contributes to AD-like brain iron dysregulation, and future research should further explore the importance of Lcn2 in AD.

Keywords: Alzheimer's disease, Lipocalin 2, Neutrophil gelatinase-associated lipocalin (NGAL), Neuroinflammation, Astrocytes, Memory, Behavior

\section{Background}

Besides aggregation of amyloid- $\beta(A \beta)$ and tau, it has become clear that Alzheimer's disease (AD) pathology is also characterized by neuroinflammation and iron dysregulation.

Neuroinflammation has been recognized to play an important role in $\mathrm{AD}$, and involves chronic activation of microglia and astrocytes in brain regions affected by $\mathrm{AD}$ pathology [1-3]. While microglia- and astrocyte-mediated immune responses may initially be neuroprotective,

\footnotetext{
* Correspondence: u.l.m.eisel@rug.nl

${ }^{2}$ Department of Molecular Neurobiology, Groningen Institute for Evolutionary Life Sciences (GELIFES), University of Groningen, Nijenborgh 7, Groningen 9747 AG, The Netherlands

${ }^{5}$ University Center of Psychiatry \& Interdisciplinary Center of Psychopathology of Emotion Regulation, University Medical Center Groningen, University of Groningen, Hanzeplein 1, Groningen 9713 GZ, The Netherlands Full list of author information is available at the end of the article
}

chronically activated brain immune cells may lose certain protective functions and acquire toxic properties $[2,4,5]$. For example, chronic production of reactive oxygen species and pro-inflammatory cytokines by activated glia contributes significantly to $\mathrm{AD}$ pathology, by activating pro-apoptotic signaling pathways and promoting further aggregation of $A \beta[6-10]$.

Iron dysregulation is also known to play an important part in AD pathology [11]. Iron accumulation occurs in brain regions affected by $\mathrm{AD}$ pathology, particularly in plaques and cell types including neurons and microglia [12-15]. Iron accumulation may contribute significantly to $\mathrm{AD}$ pathology by promoting $\mathrm{A} \beta$ aggregation, enhancing further pro-inflammatory processes, disturbing mitochondrial respiration, stimulating oxidative stress, and inducing ferroptosis $[11,16]$. 
Interestingly, findings suggest that the protein Lipocalin 2 (Lcn2) may be involved in both neuroinflammation and iron regulation, and might contribute to AD pathology. Lcn2, also known as siderocalin, 24p3, uterocalin, and neutrophil gelatinase-associated lipocalin (NGAL), is an acute-phase protein that is rapidly produced and secreted in response to a wide range of inflammatory and pathological stimuli $[17,18]$. Lcn2 plays a role in various processes, including the defense against certain bacterial infections by sequestering iron-loaded bacterial siderophores, mammalian iron metabolism, anti- and pro-inflammatory responses, anti- and pro-apoptotic signaling, and cell migration and differentiation [17, 19-21]. Studies showed that Lcn 2 protein levels in blood increase with age and mild cognitive impairment, and are increased in human post-mortem brain tissues in different diseases of the central nervous system (CNS), including AD, Parkinson's disease, and multiple sclerosis [22-27]. Studies in mice and cell culture showed that Lcn2 contributes significantly to neurodegenerative processes, for example by aggravating pro-inflammatory responses, silencing neuroprotective signaling pathways, and sensitizing brain cells to cell death [25, 27$34]$. In the context of $A D$ pathology, in vitro experiments showed that astrocytes produce high levels of Lcn2 in response to $A \beta$, and that Lcn2 renders brain cells more vulnerable to $A \beta$-induced cell death [27, 31]. Contrastingly, a few studies concerning sepsis, stroke, and multiple sclerosis reported that Lcn2 exerts significant neuroprotective functions, by promoting anti-inflammatory responses and glial prorecovery phenotypes [35-37].

Whether Lcn2 exerts significant-either neuroprotective or neurodegenerative-effects on AD pathology has not yet been studied in vivo. Therefore, the aim of the present study was to determine the effect of Lcn2 on AD-like pathology in a mouse model of AD. To this end, we compared behavior, memory functioning, and A $\beta$-associated neuropathology in J20 mice (a transgenic amyloid precursor protein (APP) overexpressing $\mathrm{AD}$ mouse model) and Lcn2-deficient J20 (J20xLcn2 KO) mice. Neuropathological investigation included analysis of hippocampal $\mathrm{A} \beta$ plaque load, activation of microglia and astrocytes, and iron load.

\section{Methods}

Mice

Male wild-type (WT), J20, Lcn2 knock-out (KO), and J20xLcn2 KO mice were studied at the behavioral and neuropathological level. Originally, the J20 AD mouse model (overexpressing human APP with the Swedish and Indiana mutations, under a PDGFB promoter [38]) was obtained from the Mutant Mouse Resource and
Research Center (MMRRC stock no. 034836-JAX; former JAX stock no. 006293), and lipocalin 2 knock-out (Lcn2 KO) mice were received from the group of Prof. Dr. T. Mak [39] (both lines are on a C57Bl/6 background). Subsequently, mice were bred in our animal facility, and J20 and Lcn2 $\mathrm{KO}$ mice were cross-bred to create a J20xLcn $2 \mathrm{KO}$ mouse line. J20 and J20xLcn2 KO mice were hemizygous for the J20 transgene, and Lcn2 $\mathrm{KO}$ and J20xLcn2 KO mice were homozygous for the Lcn2 knock-out. Genotypes of the mice were determined by polymerase chain reaction (PCR) (Additional file 1: Table S1). Behavioral experiments started when mice were 11.5 months of age. All mouse experiments performed in this study were approved by the animal ethics committee of the University of Groningen (DEC6851A).

\section{Behavioral experiments}

To study different behaviors and cognitive functions, several behavioral tests were performed, in the following order: home-cage activity measurement, open field, novel location recognition, Y-maze spontaneous alternation, elevated-plus maze, and Morris water maze. All tests were conducted by the same experimenter, who was blinded for the mouse genotypes. The arenas used for the behavioral tests (except the Morris water maze) were cleaned with ethanol in between mice. In total, 61 mice were studied, with $n=13-17$ mice per genotype (WT: $n=17$, J20: $n=13$, Lcn2 KO: $n=16$, J20xLcn2 KO: $n=15)$. Mice were studied in two cohorts of 30-31 mice, balanced for genotype. More details are included in Additional file 1.

\section{Home-cage activity}

Home-cage activity was recorded for 2 weeks for all mice, by placing each mouse cage underneath a passive infrared (PIR) sensor. Movements were aggregated and stored in one-minute bins on a computer.

\section{Open field}

For the open field test, mice were allowed to freely explore a square arena for $5 \mathrm{~min}$. Movements of the mice were tracked with specialized software (EthoVision XT version 11.5, Noldus, The Netherlands). As a measure of locomotor activity, the total distance moved was analyzed. Also, as indications of anxiety-like behavior, the time spent in the center zone of the arena and the latency to first enter the center zone were measured. More details are described in Additional file 1.

\section{Novel location recognition}

The day after the open field, the novel location recognition (NLR) test started. The NLR test is a hippocampusdependent recognition memory task, based on rodent's natural exploratory behavior and preference for novelty 
[40]. The NLR task consisted of a training and test session, $24 \mathrm{~h}$ apart. In both the NLR training and test, mice were allowed to freely explore two identical objects (placed in a square arena) for $10 \mathrm{~min}$. However, in the NLR test, one of the two objects was placed at its original (training) location while the other object was relocated. Object exploration was analyzed, and as a measure of recognition memory, the discrimination index was calculated: (time spent exploring the (to be) relocated object/total exploration time)*100. A discrimination index of above $50 \%$ indicated a preference for the relocated object. Please find more details in Additional file 1.

\section{Y-maze spontaneous alternation}

As an indication of spatial working memory, mice were studied in the Y-maze spontaneous alternation task [41]. Mice were placed in the center of a white Y-shaped maze, and allowed to freely explore all three arms for $10 \mathrm{~min}$. Trials were recorded and scored for arm entries (by a blinded observer), after which the percentage of spontaneous alternation was calculated. Also see Additional file 1.

\section{Elevated-plus maze}

To further assess anxiety-like behavior, mice were tested in the elevated-plus maze (EPM) [42]. Mice that are more anxious are likely to be more reluctant to explore open and elevated spaces. Mice were placed in the center of the EPM and were allowed to freely explore the maze for $8 \mathrm{~min}$. The crossings into open and closed arms, and the time spent in the open, closed, and center zones were scored. Also see Additional file 1.

\section{Morris water maze (hidden-platform)}

The Morris water maze (MWM) was performed to assess hippocampus-dependent spatial learning and memory [43]. The MWM was performed as described by Van Dam et al. [44, 45], with some modifications. Briefly, in the training phase, mice were trained to learn the presence and location of a platform hidden in the MWM pool. The training phase comprised of eight consecutive days, with one training block (consisting of four trials, each of maximally $120 \mathrm{~s}$ ) per mouse per day. Swimming trajectories (including escape latency and swimming distance) of the mice were tracked with EthoVision XT software (Noldus, The Netherlands). After eight MWM training days, two probe trials followed, performed $24 \mathrm{~h}$ and $48 \mathrm{~h}$ after the last training. For the probe trial, the platform was removed from the maze, and mice were allowed to swim freely for $100 \mathrm{~s}$. As a measure of spatial accuracy, the number of crossings through the original platform position was scored, as well as the time spent in the target quadrant and the other quadrants. Please find more details in Additional file 1.

\section{Immunohistochemistry for detection of $A \beta, G F A P$, Iba1,} and Lcn2

A week after the last behavioral test was finished, mice were terminally anesthetized by intraperitoneal injection with sodium pentobarbital and transcardially perfused with saline and 4\% paraformaldehyde (PFA). Brains were then post-fixed in PFA for an additional $24 \mathrm{~h}$, dehydrated, frozen, and cut in coronal sections of $20-\mu \mathrm{m}$-thick on a cryostat. Hippocampal freefloating sections were stained for $A \beta$, glial fibrillary acidic protein (GFAP) and ionized calcium binding adaptor molecule 1 (Iba1), and immunofluorescent co-stainings were performed for Lcn 2 and $A \beta$, GFAP, Iba1, and NeuN. Details of immunohistochemical stainings are included in Additional file 1.

\section{Histochemistry for detection of iron}

For detection of (mostly ferric $\mathrm{Fe}^{3+}$ ) iron, the 3,3' -diaminobenzidine (DAB)-enhanced Perls' iron stain was used. A staining protocol comparable to that reported by Chen et al. 2015 [46] was used, with some modifications, as described in Additional file 1.

\section{Quantification of (immuno)histochemical stainings}

For the $A \beta$, GFAP, and iron stainings, the coverage as well as the optical density of the staining was quantified. For analysis of the Iba1 staining, we were interested in obtaining a measure of microglial activation. To this end, the ratio between the cell body size and the total cell size of microglia was analyzed, as described previously by Hovens et al. [47, 48]. For all stainings, quantification was mostly focused on the hippocampus. The $A \beta$ staining was analyzed for the entire hippocampus. The analysis of the GFAP, Iba1, and iron stainings was performed for specific hippocampal sub-regions, including sub-regions of the cornu ammonis sector 1 (CA1) region and dentate gyrus (DG) (as indicated in the results and in Additional file 1: Figure S1). For all stainings, 3-6 hippocampi were analyzed per mouse. More details are described in Additional file 1.

\section{Statistical analysis}

Results were tested by one-way ANOVA followed by Tukey's multiple comparisons post-hoc test, or by Kruskal-Wallis test followed by Dunn's multiple comparisons test when data was not normally distributed or variances were unequal. Two-way repeated measures ANOVA was used to analyze the MWM training phase. Statistical analysis was performed using GraphPad Prism version 5.0 for Windows. Data are presented as mean \pm standard error of the mean (SEM). Differences between Lcn2 KO mice and J20/J20xLcn2 KO mice were not indicated, since comparisons between these groups were 
not considered relevant to our study aims. Differences were considered statistically significant when $p<0.05$.

\section{Results}

J20 and J20xLCn2 KO mice are equally hyperactive

Home-cage activity of WT, J20, Lcn2 KO, and J20xLcn2 KO mice (11.5 months of age) was recorded for 2 weeks. Home-cage activity measurement revealed clear hyperactivity in J20 and J20xLcn2 KO mice $(p<0.0001$ and $p<0.05$ respectively, compared to WT, Additional file 1: Figure S2A, B), corresponding to hyperactive behavior in the open field $(p<0.05$ compared to WT, Additional file 1: Figure S2C) and in other tests (Additional file 1: Figure. S3). Although the home-cage activity results seem to suggest that J20xLcn2 KO mice might be less hyperactive as J20 mice, this difference was not statistically significant. Thus, it appears that J20 and J20xLcn2 KO mice show clear and equally strong hyperactivity.

Another important basic behavioral characteristic, anxiety-like behavior, was studied in the open field and elevated-plus maze (EPM). Despite an increased time spent in the center zone of the open field in Lcn2 KO mice compared to WT mice $(p<0.05$, Additional file 1 : Figure S4A), no differences were found in the latency to enter the center zone of the open field (Additional file 1: Figure $\mathrm{S4B}$ ), in the time spent on the open or closed arms in the EPM (Additional file 1: Figure S4C, D) or in the number of EPM arm entries (data not shown). Thus, overall, there seem to be no significant differences in anxiety-like behavior between the studied genotypes.

\section{J20 and J20xLcn2 KO mice show equally severe memory impairment}

Next, we aimed to assess whether the lack of Lcn 2 in J20xLcn2 KO mice caused a difference in working memory compared to J20 mice in the Y-maze spontaneous alternation task. As shown in Fig. 1a, no differences in working memory were found between the studied genotypes. Also, no significant differences were found for the number of arm entries in the Y-maze spontaneous alternation (Fig. 1b).

Subsequently, hippocampus-dependent memory functioning was investigated in the novel location recognition (NLR) task and the hidden-platform Morris water maze (MWM). In the training of the NLR, as expected, none of the groups showed a preference for one of the two identical objects that had been placed in the open field (Fig. 1c, showing a discrimination index of around 50\%). In the NLR test (24 $\mathrm{h}$ after the training), both WT and Lcn2 KO mice spent more time exploring the relocated object, indicating intact recognition memory (discrimination indices were significantly above chance level (50\%), $p<0.05$ and $p$ $<0.01$, respectively, Fig. 1d). Lcn2 KO mice did not differ from WT mice in recognition memory. J20 and J20xLcn2 $\mathrm{KO}$ mice performed significantly worse compared to WT mice $(p<0.01$ and $p<0.05$, respectively) and showed a discrimination index that was not significantly different from chance level, indicating impaired recognition memory. No significant difference between J20 and J20xLcn2 KO mice was observed.

In the MWM J20 and J20xLcn2 KO mice presented impaired learning compared to WT mice, as seen from the reduced slope of the learning curves of the escape latency and swim distance parameters $(p<0.01$ and $p<0.0001$ respectively, Fig. 1e, f). Also in the probe trials, performed $24 \mathrm{~h}$ and $48 \mathrm{~h}$ after the last MWM training, J20 and J20xLcn2 KO had significantly worse memory of the platform position and the target quadrant (Fig. 1g, h, and Additional file 1: Figure S5), as seen from fewer crossings over the position where the platform used to be, and less time spent in the target quadrant $(p<0.05)$.

Thus, J20 and J20xLcn2 KO mice showed equally severe memory impairment in both the NLR test and the MWM. Of note, Lcn2 KO mice showed significantly slower MWM learning curves compared to WT mice, possibly indicating mild memory impairment during the training phase $(p<0.05$, Fig. 1e, f). However, Lcn2 KO mice performed similar to WT mice in the first and second probe trial (Fig. 1g, h and, Additional file 1: Figure S5).

\section{J20 mice show increased hippocampal Lcn2 levels in astrocytes}

To confirm that Lcn2 levels are increased in the brains of J20 mice, and to assess the cell type to which Lcn2 was mainly localized, fluorescent stainings were performed. As shown in Fig. 2a, clear Lcn2-positive cells are indeed present in the J20 mouse brain, while WT mouse brains only show faint Lcn2-positive staining. To assess the brain cell type in J20 mouse brains in which Lcn2 was localized, co-stainings were performed for Lcn2 and NeuN, Iba1, and GFAP (Fig. 2b-d). Lcn2-positive staining did not co-localize with the neuronal marker NeuN or the microglia marker Iba1, but did clearly co-localize with GFAP-positive astrocytes.

\section{J20 and J20xLCn2 KO mice develop similar hippocampal plaque load, and show equal microglia and astrocyte activation}

Although no differences were found between J20 and J20xLcn2 KO mice in behavioral and memory phenotype, it is possible that differences may be present at the neuropathological level. To investigate this, hippocampal slices were stained for (1) A $\beta$ to assess potential differences in $A \beta$ accumulation, (2) GFAP to assess possible differences in astrocyte activity, (3) Iba1 to determine 


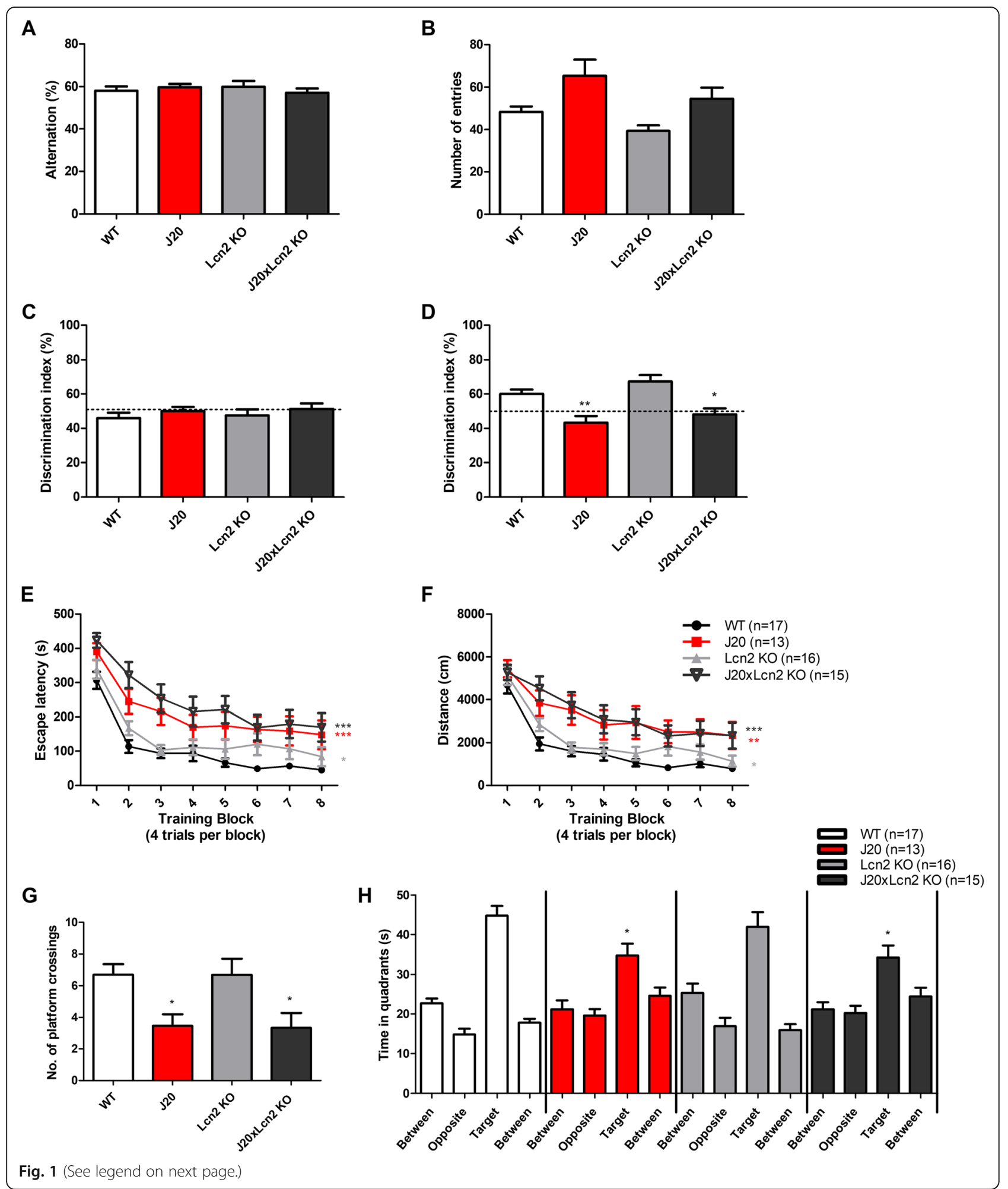


(See figure on previous page.)

Fig. 1 Impaired hippocampus-dependent long-term memory in J20 and J20xLcn2 KO mice, no differences in working memory. a Alternation (\%) in the Ymaze spontaneous alternation test. $\mathbf{b}$ Number of arm entries in the $Y$-maze spontaneous alternation test (10 min). Tested with one-way ANOVA with Tukey's multiple comparisons test. $n=13-17$ mice per group (WT $n=17, \mathrm{~J} 20 n=13$, Lcn2 KO $n=16, J 20 x \mathrm{Lcn} 2 \mathrm{KO} n=15$ ). c Discrimination index (\%) in the novel location recognition (NLR) training and $\mathbf{d}$ in the NLR test, that was performed $24 \mathrm{~h}$ after the NLR training. Dashed line indicates $50 \%$ chance level. Of note, one Lcn2 KO and one J20xLcn2 KO mouse had to be excluded from the analysis due to lack of exploratory behavior. $n=13-17$ mice per group (WT $n=17, \mathrm{~J} 20 n=13, \mathrm{Lcn} 2 \mathrm{KO} n=15, \mathrm{~J} 20 \mathrm{xLCn} 2 \mathrm{KO} n=14$ ). Tested with one-way ANOVA with Tukey's multiple comparisons test. e Escape latency (s) and (f) swim distance (cm) in the training phase of the Morris water maze (MWM). $n=13-17$ mice per group (WT $n=17, J 20 n=13, \operatorname{Lcn} 2 \mathrm{KO} n=16, J 20 \times \mathrm{Lcn} 2 \mathrm{KO}$ $n=15)$. Tested with two-way repeated measures ANOVA. $\mathbf{g}$ Number of platform crossings and $\mathbf{h}$ time spent in the pool quadrants during the probe trial (24 h after the last training trial). $n=13-17$ mice per group ( $\mathrm{WT} n=17, \mathrm{~J} 20 n=13, \mathrm{Lcn} 2 \mathrm{KO} n=16, \mathrm{~J} 20 \mathrm{xLcn} 2 \mathrm{KO} n=15)$. Tested with one-way ANOVA with Tukey's multiple comparisons test. ${ }^{*} p<0.05 ;{ }^{* *} p<0.01$; ${ }^{* * *} p<0.0001$ compared to WT. No significant differences were present between J20 and J20xLcn2 KO mice

possible differences in microglia activation, and (4) iron to investigate potential differences in iron accumulation. As shown in Fig. 3a, clear $A \beta$ aggregation and plaque load was present in the hippocampus of J20 mice, quantified by measuring the total coverage of $\mathrm{A} \beta$-staining ( $p$ $<0.0001$ compared to WT). J20xLcn2 KO mice, however, showed a similar hippocampal coverage of $\mathrm{A} \beta$-positive staining. Clear astrogliosis $(p<0.0001$ increase in GFAP coverage compared to WT) and microglia activation $(p<0.01$ increase in microglia activity compared to WT) were also found in the hippocampus of J20 mice, yet, equal glial activation was found in

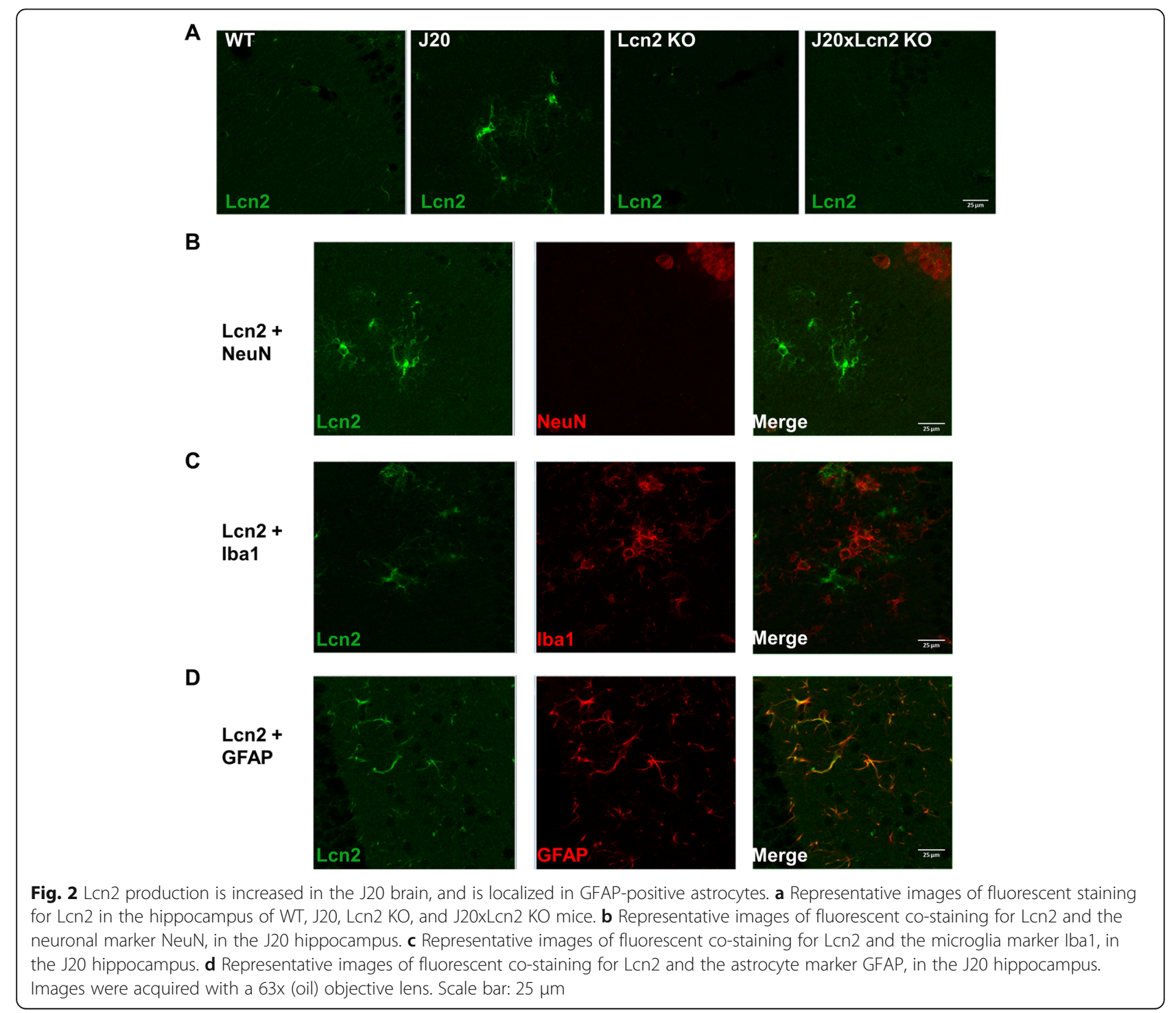




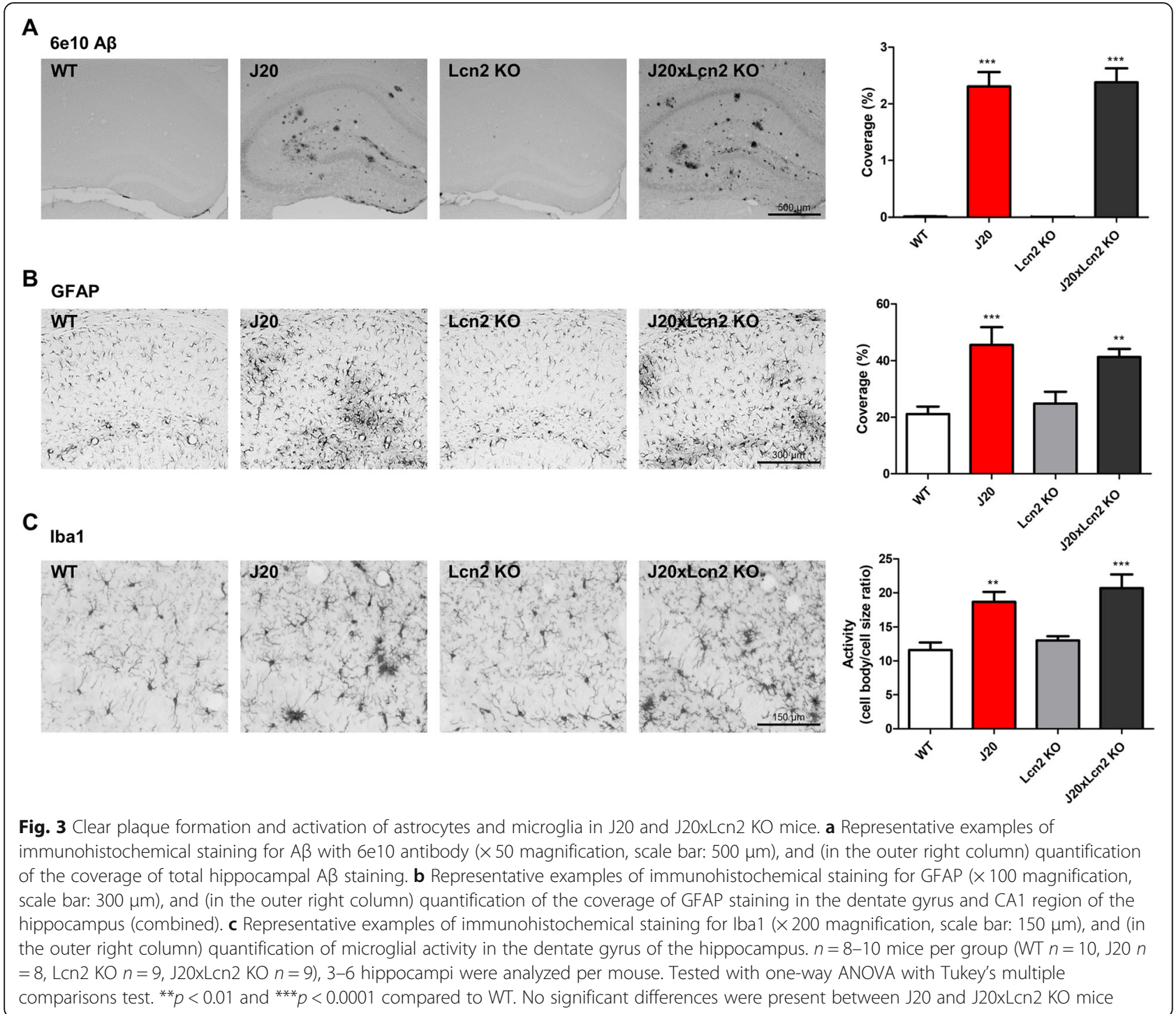

J20xLcn2 KO mice (Fig. 3b, c). Astrocyte and microglia activation were assessed in specific hippocampal sub-regions. Interestingly, astrocyte activation in J20 and J20xLcn2 KO mice appeared to be induced equally strongly in all hippocampal sub-regions analyzed, as well as the corpus callosum (Additional file 1: Figure S6). In contrast, it appeared that microglia activation may depend more on the specific hippocampal region: while clear microglia activation is found in the dentate gyrus, microglia activation is not significantly increased in the CA1 region (Additional file 1: Figure S7).

Iron dysregulation is present in the hippocampus of $\mathrm{J} 20$ mice, and is less severe in J20xLcn2 KO mice

Finally, potential differences in hippocampal iron regulation were investigated between genotypes. To this end, hippocampal sections were stained by DABenhanced Perls' staining (detecting mostly ferric $\mathrm{Fe}^{3+}$ iron), and the coverage and optical density of iron staining were analyzed in different hippocampal sub-regions. As shown in Fig. 4a, a clear increase in iron staining was detected in the hippocampus of J20 mice, including both plaque-like iron staining and more diffuse and intracellular iron staining (such as the staining seen in the pyramidal and granular neuronal layers in the CA1 and dentate gyrus). As shown in Fig. 4b, c and Additional file 1: Figure S8A, B, quantification of the coverage and optical density of the iron-positive staining confirmed this observation of increased iron staining in $\mathrm{J} 20$ mice as compared to WT mice, in many of the studied hippocampal sub-regions $(p<0.05$ or $p<0.01$ in different hippocampal sub-regions). Intriguingly, less iron staining is visible in the hippocampus of J20xLcn2 KO mice, as compared to J20 mice ( $p<0.05$ in different hippocampal sub-regions). Moreover, it appeared that Lcn2 KO 


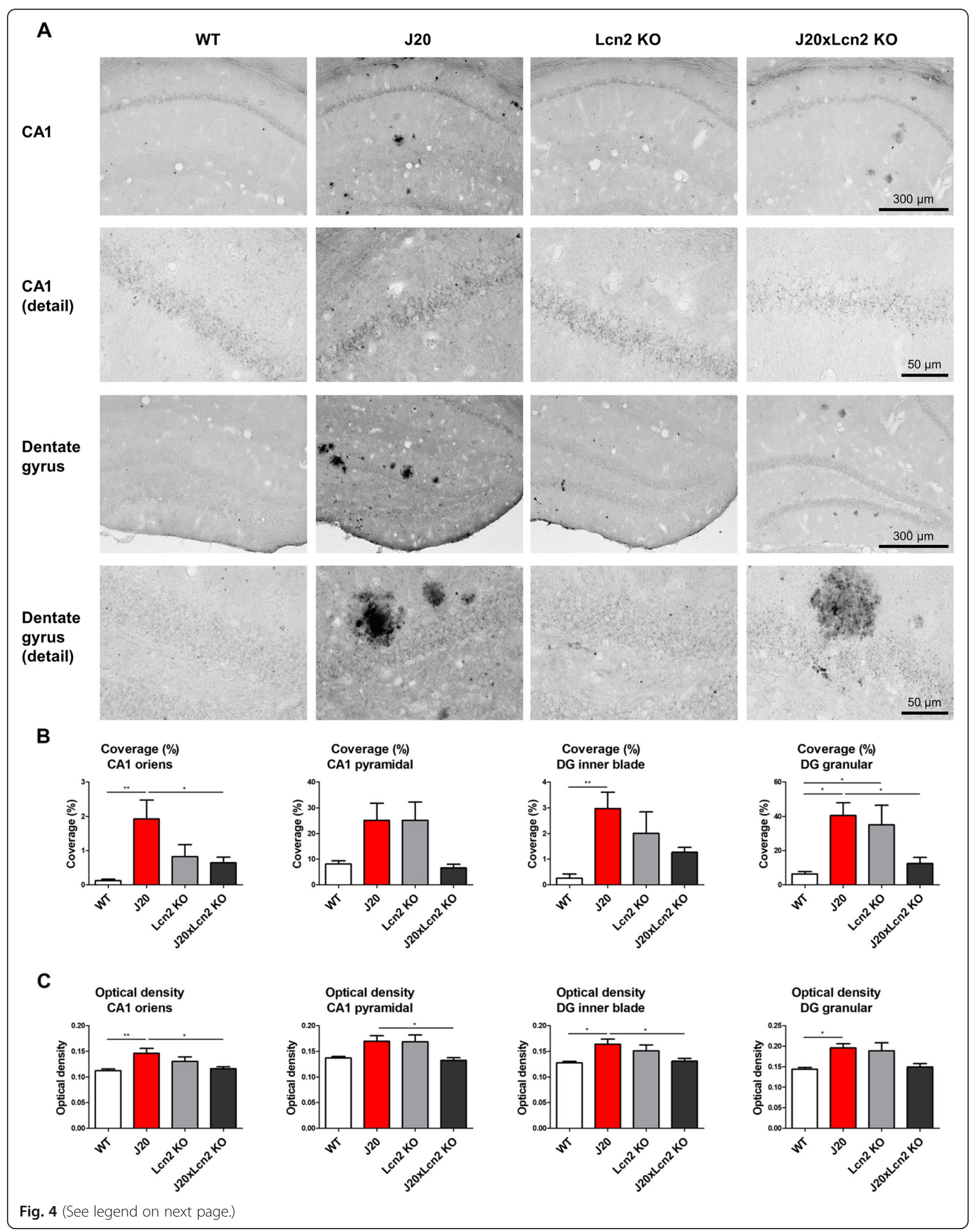


(See figure on previous page.)

Fig. 4 Increased hippocampal iron accumulation in J20 mice, and less severe iron accumulation in J20xLcn2 KO mice. a Representative examples of histochemical staining for iron by enhanced Perls' reaction. Images were acquired and analysis was performed at $\times 100$ magnification (scale bar: $300 \mu \mathrm{m}$ ). Detailed images were acquired at $\times 400$ magnification (scale bar: $50 \mu \mathrm{m}$ ). Examples include a focus on the CA1 and the dentate gyrus regions of the hippocampus. $\mathbf{b}$ Quantification of the coverage of iron-positive staining, in the stratum oriens and pyramidal layer of the CA1 region, and in the inner blade and granular layer of the dentate gyrus. Of note, due to regional differences in intensity of the iron staining, the iron staining in the CA1 pyramidal and DG granular cell layers was analyzed with a different detection threshold than the detection threshold used to analyze the other hippocampal sub-regions. This explains the higher relative coverages of iron staining found for the CA1 pyramidal and DG granular cell layers, as compared to other hippocampal sub-regions. c Quantification of the optical density of iron-positive staining, in the stratum oriens and pyramidal layer of the CA1 region, and in the inner blade and granular layer of the dentate gyrus. $n=8-9$ mice per group (WT $n=9, J 20 n=8, \operatorname{Lcn} 2 \mathrm{KO} n=9$, J20xLcn2 KO $n=9$ ), 3-4 hippocampi were analyzed per mouse. Tested with Kruskal-Wallis test with Dunn's multiple comparisons post-hoc test. ${ }^{*} p<0.05$ and ${ }^{* *} p<0.01$

mice may display stronger hippocampal iron staining as compared to WT mice, in different hippocampal sub-regions.

\section{Discussion}

We report that J20 and Lcn2-deficient J20 (J20xLcn2 KO) mice at 12 months of age show equally severe AD-like behavioral changes, cognitive impairment, plaque formation, and glial activation. Intriguingly, J20xLcn2 KO mice presented significantly decreased AD-like iron accumulation in the hippocampus, as compared to J20 mice.

The reduced hippocampal iron load in J20xLcn2 KO versus $\mathrm{J} 20$ mice indicates that $\mathrm{Lcn} 2$ contributes to AD-like iron accumulation in the brain. This result corresponds with the finding of Lcn2-mediated brain iron accumulation in an animal model of hemorrhagic stroke [34]. Also, Lcn2 was reported to stimulate ferritin mRNA expression in $A \beta$-treated cultured astrocytes, indicating a Lcn2-mediated increase in iron storage upon $\mathrm{A} \beta$-challenge [31].

Lcn 2 is known to be involved in iron metabolism by binding to bacterial and mammalian siderophores (small iron-binding molecules) present in the body [49-51]. Lcn 2 is able to mediate both import and export of iron into/from cells $[52,53]$. Under inflammatory conditions, Lcn 2 contributes to an iron-retentive response known as the anemia of inflammation, by promoting iron retention in macrophages $[54,55]$. Possibly a similar iron-retentive response occurs in the inflamed brain. Indeed, astrocytes, neurons, and especially microglia (the macrophages of the brain) were found to accumulate high levels of non-transferrin bound iron upon inflammatory stimulation [15, 56-61], which might possibly in part be regulated by $\operatorname{Lcn} 2$.

This possibility may explain the observed Lcn2mediated iron retention in J20 versus J20xLcn2 KO brains, and warrants further investigation of involved cell types and siderophores. Moreover, the pathophysiological relevance of Lcn2-mediated brain iron accumulation in $\mathrm{AD}$ requires further research. Namely, despite the well-known important role of iron dysregulation in different AD-related pathophysiological processes, the alleviated iron accumulation in J20xLcn2 $\mathrm{KO}$ mice as compared to J20 mice is not accompanied by significant changes in other AD characteristics, such as behavior, cognition, $A \beta$ aggregation, and glial activation. It may be possible that the hippocampal iron accumulation in 12-month-old J20 mice is not severe enough (yet) to overwhelm the stable iron storage facilities in the brain, and/or that other compensatory mechanisms induced upon iron dysregulation are (still) sufficient to protect the brain from iron toxicity. This scenario would explain why the difference in iron load between J20 and J20xLcn2 KO mice does not result in significant differences in other $A D$ characteristics. It would be of great interest to study this hypothesis, including the possibility that (Lcn2-mediated) iron dysregulation may become more severe in more advanced $\mathrm{AD}$ mice. In these future studies, it would be interesting to for example assess potential differences in levels of labile reactive iron, oxidative stress, and ferroptosis.

Interestingly, the results suggests that Lcn2 may also affect iron homeostasis under healthy unchallenged conditions, as indicated by the increased hippocampal iron levels in Lcn2 $\mathrm{KO}$ mice as compared to WT mice. It should be noted that significance between WT and Lcn2 $\mathrm{KO}$ mice was often not reached when all four genotypes were compared, while significant differences were found when only WT and Lcn2 KO mice were compared. This finding corresponds with previous reports, in which increased iron levels were found in macrophages and neural stem cells in healthy Lcn2 KO mice [62-64].

Taken together, basal physiological levels of Lcn2 under healthy conditions may be required to maintain iron homeostasis (with absence of Lcn2 leading to iron accumulation in certain cell types, possibly by reduced iron export), while increased Lcn2 levels under pathological conditions may contribute to iron dysregulation and accumulation as well (with increased Lcn2 levels leading to iron accumulation in plaques and certain cell types, possibly by increased iron import). Importantly, although iron homeostasis is tightly regulated, it thus appears that Lcn2 has an important function in iron regulation and distribution in the brain. 
No differences were detected in behavior, cognition, plaque load, and glial activation between J20 and J20xLcn2 KO mice. The absence of significant detrimental or beneficial effects of Lcn2 on these AD-like characteristics is notable, taking into account the strong neurodegenerative $[22,25,27-29,31-34,65-69]$ and neuroprotective [35-37] effects that were reported for Lcn2 in various in vitro and animal models of brain injury and disease. It is possible that Lcn2 indeed does not affect cognition and glial activation in $\mathrm{AD}$, which would provide interesting contrasting information on the role of Lcn2 in neurodegeneration. Alternatively, it is possible that Lcn2 might affect AD pathology in other models of AD. For example, it would be important to validate if Lcn2 might affect cognitive function and pathology in AD models that present more severe neuronal loss (for example APP/PS1KI mice [70]), or in mice at other ages. Furthermore, although clear Lcn2-positive astrocytes were detected, it should be determined whether Lcn2 in the J20 mouse brain reaches concentrations that are comparable to the Lcn2 levels in the human AD brain. Related to this, it would be of interest to investigate $\mathrm{AD}$ models which overexpress Lcn2 (besides Lcn2-deficient AD models). Of note, most studies regarding the role of Lcn2 in CNS disease/injury until now were performed in acute models of CNS injury (which may cause stronger, short-term, induction of Lcn2), in contrast to the chronic transgenic AD model studied here (in which-in comparison to models of acute injury-Lcn 2 expression may be less strong, which may also be the case for the expression of other inflammatory mediators such as interleukin 1 beta (IL-1 $\beta$, also see, Additional file 1: Figure S9)). Finally, it should be taken into account that $\mathrm{J} 20$ mice overexpress human $A \beta$ while expressing murine Lcn2 (62\% identical to human Lcn2 on the amino acid level [71]), which might impact their potential direct or indirect interactions and the translational value to the human disease.

Taken together, more insight into the protective and toxic effects of Lcn2 is required to understand its importance in $\mathrm{AD}$, and its potential as a therapeutic target. Of note, while Lcn $2 \mathrm{KO}$ mice are of great value in the study of Lcn2, it should be considered that potential mild developmental disturbances may be present in these mice. As shown in the current and previous studies $[62,63]$, healthy unchallenged Lcn2 KO mice may display disturbed iron regulation in the periphery and the brain, which might affect important physiological processes such as synaptic plasticity and neurogenesis [62, 72, 73]. Possibly related to this, Lcn2 KO mice appear to present a mild cognitive impairment, as seen from the MWM learning curves in Fig. 3e, $\mathrm{f}$ (Additional file 1: Figure S10) and previous reports $[62,72]$. Interestingly, this mild cognitive impairment might in part be overcome with age (Additional file 1: Figure S10). To circumvent potential mild developmental disturbances in Lcn $2 \mathrm{KO}$ mice, it would be of interest to investigate conditional Lcn2 $\mathrm{KO}$ (or conditional Lcn2-overexpressing) mice.

\section{Conclusions}

The results from this study show that Lcn2 contributes to hippocampal iron accumulation in the J20 AD mouse model. As such, this study provides new insights into Lcn2 as a potential functional element in iron dysregulation in the AD brain. Lcn2 did not significantly affect other AD-like characteristics (including behavioral changes, cognitive impairment, plaque load, and glial activation) in the J20 mouse model at 12 months of age. However, it is possible that such effects might surface in other AD models and/or at other ages, which should be investigated in future work.

\section{Additional file}

Additional file 1: Lipocalin 2 contributes to brain iron dysregulation but does not affect cognition, plaque load and glial activation in the J20 Alzheimer mouse model. Contains supplementary information and supplementary figures, belonging to the study 'Lipocalin 2 contributes to brain iron dysregulation but does not affect cognition, plaque load and glial activation in the J20 Alzheimer mouse model'. (PDF 1866 kb)

\section{Abbreviations}

AD: Alzheimer's disease; APP: Amyloid precursor protein; A $\beta$ : Amyloid- $\beta$; CA1: Cornu ammonis sector 1; CNS: Central nervous system; DAB: 3,3'Diaminobenzidine; DG: Dentate gyrus; EPM: Elevated-plus maze; $\mathrm{Fe}^{3+}$ : Ferric iron; GFAP: Glial fibrillary acidic protein; Iba1: Ionized calcium binding adaptor molecule 1; Len2 KO: Lipocalin 2 knock-out; Len2: Lipocalin 2; MWM: Morris water maze; NGAL: Neutrophil gelatinase-associated lipocalin; NLR: Novel location recognition; PCR: Polymerase chain reaction; WT: Wild type

\section{Acknowledgments}

We thank Wendy Kaspers, Roelie Veenstra-Wiegman, Benjamin Otten, and Diane ten Have for their fantastic work in mouse breeding and animal caretaking, and Wanda Douwenga and Kunja Slopsema for excellent technical assistance. We also thank Dr. Debby Van Dam and Elly Geerts from the University of Antwerp for very helpful advice on Morris water maze set-up, and Klaas Sjollema for excellent technical assistance with imaging of immunofluorescent stainings at the UMCG Microscopy and Imaging Center (UMIC).

\section{Funding}

This study was funded by a grant from the Internationale Stichting Alzheimer Onderzoek (ISAO grant \#06511 to ULME and PPDD). Furthermore, this work was supported by ZonMW Deltaplan Dementie Memorabel (grant to PJWN, PPDD and ULME), a research grant of the Interuniversity Poles of Attraction (IAP Network P7/16) of the Belgian Federal Science Policy Office, Methusalem excellence grant of the Flemish Government, the Thomas Riellaerts research fund, University Research Fund of the University of Antwerp, NeuroSearch Antwerp, and the Alzheimer Research Center Groningen (grants to PPDD). DWD is funded by a grant of the Research School of Behavioral and Cognitive Neurosciences (BCN) and a grant from Stichting Hadders-De Cock (2017-30), and PJWN is funded by Alzheimer Nederland (WE. 13-2015-19) and NeuroSearch Antwerp.

\section{Availability of data and materials}

The datasets used and/or analyzed during the current study are available from the corresponding author on reasonable request. 


\section{Authors' contributions}

DWD, PJWN, ASB, PPDD, and ULME designed the study and interpreted the results. DWD performed experiments, analyzed data, and wrote the manuscript. PJWN contributed to writing the manuscript, and PJWN and ASB contributed to data analysis. JNK prepared microscope settings for microscopy analyses. All authors contributed to editing of the manuscript. All authors read and approved the final manuscript.

\section{Ethics approval}

All applicable international, national, and/or institutional guidelines for the care and use of animals were followed. All procedures performed in studies involving animals were in accordance with EU regulations (EU Directive 2010/63/EU for animal experiments), and were approved of by the ethical animal experiment committee and the animal welfare body of the University of Groningen (Groningen, The Netherlands; DEC6851A).

\section{Consent for publication}

Not applicable.

\section{Competing interests}

The authors declare that they have no competing interests.

\section{Publisher's Note}

Springer Nature remains neutral with regard to jurisdictional claims in published maps and institutional affiliations.

\section{Author details \\ 'Department of Neurology and Alzheimer Research Center, University Medical Center Groningen, University of Groningen, Hanzeplein 1, Groningen 9713 GZ, The Netherlands. 'Department of Molecular Neurobiology, Groningen Institute for Evolutionary Life Sciences (GELIFES), University of Groningen, Nijenborgh 7, Groningen 9747 AG, The Netherlands. ${ }^{3}$ Department of Nuclear Medicine and Molecular Imaging, University Medical Center Groningen, University of Groningen, Hanzeplein 1, Groningen 9713 GZ, The Netherlands. ${ }^{4}$ Laboratory of Neurochemistry and Behavior, Biobank, Institute Born-Bunge, University of Antwerp, Universiteitsplein 1, 2610 Antwerp, Belgium. ${ }^{5}$ University Center of Psychiatry \& Interdisciplinary Center of Psychopathology of Emotion Regulation, University Medical Center Groningen, University of Groningen, Hanzeplein 1, Groningen 9713 GZ, The Netherlands.}

\section{Received: 24 July 2018 Accepted: 18 November 2018}

\section{Published online: 30 November 2018}

\section{References}

1. Akiyama H, Barger S, Barnum S, Bradt B, Bauer J, Cole GM, et al. Inflammation and Alzheimer's disease. Neurobiol Aging. 2000;21:383-421.

2. Heneka MT, Carson MJ, El Khoury J, Landreth GE, Brosseron F, Feinstein DL, et al. Neuroinflammation in Alzheimer's disease. Lancet Neurol. 2015;14:388405. https://doi.org/10.1016/S1474-4422(15)70016-5.

3. Eikelenboom P, Veerhuis R, Scheper W, Rozemuller AJM, van Gool WA, Hoozemans JJM. The significance of neuroinflammation in understanding Alzheimer's disease. J Neural Transm (Vienna). 2006;113:1685-95. https://doi. org/10.1007/s00702-006-0575-6

4. Meraz-Ríos MA, Toral-Rios D, Franco-Bocanegra D, Villeda-Hernández 」, Campos-Peña V. Inflammatory process in Alzheimer's disease. Front Integr Neurosci. 2013;7:59. https://doi.org/10.3389/fnint.2013.00059.

5. Verkhratsky A, Olabarria M, Noristani HN, Yeh C-Y, Rodriguez JJ. Astrocytes in Alzheimer's disease. Neurother J Am Soc Exp Neurother. 2010;7:399-412. https://doi.org/10.1016/j.nurt.2010.05.017.

6. Combs CK, Karlo JC, Kao SC, Landreth GE. Beta-amyloid stimulation of microglia and monocytes results in TNFalpha-dependent expression of inducible nitric oxide synthase and neuronal apoptosis. J Neurosci. 2001;21:1179-88.

7. He P, Zhong Z, Lindholm K, Berning L, Lee W, Lemere C, et al. Deletion of tumor necrosis factor death receptor inhibits amyloid beta generation and prevents learning and memory deficits in Alzheimer's mice. J Cell Biol. 2007; 178:829-41. https://doi.org/10.1083/jcb.200705042.

8. Kitazawa M, Oddo S, Yamasaki TR, Green KN, LaFerla FM. Lipopolysaccharide-induced inflammation exacerbates tau pathology by a cyclin-dependent kinase 5-mediated pathway in a transgenic model of
Alzheimer's disease. J Neurosci. 2005;25:8843-53. https://doi.org/10.1523/ JNEUROSCI.2868-05.2005.

9. Metcalfe MJ, Figueiredo-Pereira ME. Relationship between tau pathology and Neuroinflammation in Alzheimer's disease. Mt Sinai J Med N Y. 2010;77: 50-8. https://doi.org/10.1002/msj.20163.

10. Zhao J, O'Connor T, Vassar R. The contribution of activated astrocytes to $A B$ production: implications for Alzheimer's disease pathogenesis. J Neuroinflammation. 2011;8:150. https://doi.org/10.1186/1742-2094-8-150.

11. Belaidi AA, Bush Al. Iron neurochemistry in Alzheimer's disease and Parkinson's disease: targets for therapeutics. J Neurochem. 2016;139(Suppl 1):179-97. https://doi.org/10.1111/jnc.13425.

12. LeVine SM. Iron deposits in multiple sclerosis and Alzheimer's disease brains. Brain Res. 1997;760:298-303. https://doi.org/10.1016/S0006-8993(97)00470-8.

13. van Duijn S, Bulk M, van Duinen SG, Nabuurs RJA, van Buchem MA, van der Weerd $L$, et al. Cortical iron reflects severity of Alzheimer's disease. J Alzheimers Dis JAD. 2017;60:1533-45. https://doi.org/10.3233/JAD-161143.

14. Sands SA, Leung-Toung R, Wang Y, Connelly J, LeVine SM. Enhanced histochemical detection of Iron in paraffin sections of mouse central nervous system tissue: application in the APP/PS1 mouse model of Alzheimer's disease. ASN Neuro. 2016;8:1759091416670978. https://doi.org/ 10.1177/1759091416670978.

15. Holland R, Mclntosh AL, Finucane OM, Mela V, Rubio-Araiz A, Timmons G, et al. Inflammatory microglia are glycolytic and iron retentive and typify the microglia in APP/PS1 mice. Brain Behav Immun. 2018;68:183-96. https://doi. org/10.1016/j.bbi.2017.10.017

16. Morris G, Berk M, Carvalho AF, Maes M, Walker AJ, Puri BK. Why should neuroscientists worry about iron? The emerging role of ferroptosis in the pathophysiology of neuroprogressive diseases. Behav Brain Res. 2018;341: 154-75. https://doi.org/10.1016/j.bbr.2017.12.036.

17. Chakraborty S, Kaur S, Guha S, Batra SK. The multifaceted roles of neutrophil gelatinase associated lipocalin (NGAL) in inflammation and cancer. Biochim Biophys Acta. 1826;2012:129-69. https://doi.org/10.1016/j.bbcan.2012.03.008.

18. Flower DR. The lipocalin protein family: structure and function. Biochem J. 1996;318:1-14.

19. Jha MK, Lee S, Park DH, Kook H, Park K-G, Lee I-K, et al. Diverse functional roles of lipocalin-2 in the central nervous system. Neurosci Biobehav Rev. 2015;49:135-56. https://doi.org/10.1016/j.neubiorev.2014.12.006.

20. Ferreira AC, Dá Mesquita S, Sousa JC, Correia-Neves M, Sousa N, Palha JA, et al. From the periphery to the brain: Lipocalin-2, a friend or foe? Prog Neurobiol. 2015;131:120-36. https://doi.org/10.1016/j.pneurobio.2015.06.005.

21. Flo TH, Smith KD, Sato S, Rodriguez DJ, Holmes MA, Strong RK, et al. Lipocalin 2 mediates an innate immune response to bacterial infection by sequestrating iron. Nature. 2004;432:917-21. https://doi.org/10.1038/nature03104.

22. Al Nimer F, Elliott C, Bergman J, Khademi M, Dring AM, Aeinehband S, et al. Lipocalin-2 is increased in progressive multiple sclerosis and inhibits remyelination. Neurol Neuroimmunol Neuroinflammation. 2016;3:e191. https://doi.org/10.1212/NXI.0000000000000191.

23. Choi J, Lee H-W, Suk K. Increased plasma levels of lipocalin 2 in mild cognitive impairment. J Neurol Sci. 2011;305:28-33. https://doi.org/10.1016/j. jns.2011.03.023.

24. Dekens DW, Naudé PJW, Engelborghs S, Vermeiren Y, Van Dam D, Oude Voshaar RC, et al. Neutrophil gelatinase-associated lipocalin and its receptors in Alzheimer's disease (AD) brain regions: differential findings in AD with and without depression. J Alzheimers Dis JAD. 2017;55:763-76. https://doi.org/10.3233/JAD-160330.

25. Kim B-W, Jeong KH, Kim J-H, Jin M, Kim J-H, Lee M-G, et al. Pathogenic upregulation of glial lipocalin-2 in the parkinsonian dopaminergic system. J Neurosci. 2016;36:5608-22. https://doi.org/10.1523/JNEUROSCI.4261-15.2016.

26. Naudé PJW, Eisel ULM, Comijs HC, Groenewold NA, De Deyn PP, Bosker FJ, et al. Neutrophil gelatinase-associated lipocalin: a novel inflammatory marker associated with late-life depression. J Psychosom Res. 2013;75:44450. https://doi.org/10.1016/j.jpsychores.2013.08.023.

27. Naudé PJW, Nyakas C, Eiden LE, Ait-Ali D, van der Heide R, Engelborghs S, et al. Lipocalin 2: novel component of proinflammatory signaling in Alzheimer's disease. FASEB J Off Publ Fed Am Soc Exp Biol. 2012;26:2811-23. https://doi.org/10.1096/fj.11-202457.

28. Jin M, Jang E, Suk K. Lipocalin-2 acts as a neuroinflammatogen in lipopolysaccharide-injected mice. Exp Neurobiol. 2014;23:155-62. https://doi. org/10.5607/en.2014.23.2.155

29. Kim J-H, Ko P-W, Lee H-W, Jeong J-Y, Lee M-G, Kim J-H, et al. Astrocytederived lipocalin-2 mediates hippocampal damage and cognitive deficits in 
experimental models of vascular dementia. Glia. 2017;65:1471-90. https:// doi.org/10.1002/glia.23174.

30. Lee S, Jha MK, Suk K. Lipocalin-2 in the inflammatory activation of brain astrocytes. Crit Rev Immunol. 2015;35:77-84.

31. Mesquita SD, Ferreira AC, Falcao AM, Sousa JC, Oliveira TG, Correia-Neves M, et al. Lipocalin 2 modulates the cellular response to amyloid beta. Cell Death Differ. 2014;21:1588-99. https://doi.org/10.1038/cdd.2014.68.

32. Nam Y, Kim J-H, Seo M, Kim J-H, Jin M, Jeon S, et al. Lipocalin-2 protein deficiency ameliorates experimental autoimmune encephalomyelitis the pathogenic role of lipocalin-2 in the central nervous system and peripheral lymphoid tissues. J Biol Chem. 2014;289:16773-89. https://doi.org/10.1074/ jbc.M113.542282

33. Wang G, Weng Y-C, Han X, Whaley JD, McCrae KR, Chou W-H. Lipocalin-2 released in response to cerebral ischaemia mediates reperfusion injury in mice. J Cell Mol Med. 2015;19:1637-45. https://doi.org/10.1111/jcmm.12538.

34. Ni W, Zheng M, Xi G, Keep RF, Hua Y. Role of lipocalin-2 in brain injury after intracerebral hemorrhage. J Cereb Blood Flow Metab Off J Int Soc Cereb Blood Flow Metab. 2015;35:1454-61. https://doi.org/10.1038/jcbfm.2015.52.

35. Berard JL, Zarruk JG, Arbour N, Prat A, Yong WW, Jacques FH, et al. Lipocalin 2 is a novel immune mediator of experimental autoimmune encephalomyelitis pathogenesis and is modulated in multiple sclerosis. Glia. 2012;60:1145-59. https://doi.org/10.1002/glia.22342.

36. Kang SS, Ren Y, Liu C-C, Kurti A, Baker KE, Bu G, et al. Lipocalin-2 protects the brain during inflammatory conditions. Mol Psychiatry. 2018;23:344-50. https://doi.org/10.1038/mp.2016.243.

37. Xing C, Wang X, Cheng C, Montaner J, Mandeville E, Leung W, et al. Neuronal production of lipocalin-2 as a help-me signal for glial activation. Stroke. 2014;45:2085-92. https://doi.org/10.1161/STROKEAHA.114.005733.

38. Mucke L, Masliah E, Yu G-Q, Mallory M, Rockenstein EM, Tatsuno G, et al. High-level neuronal expression of A $1-42$ in wild-type human amyloid protein precursor transgenic mice: Synaptotoxicity without plaque formation. J Neurosci. 2000;20:4050-8.

39. Berger T, Togawa A, Duncan GS, Elia AJ, You-Ten A, Wakeham A, et al. Lipocalin 2-deficient mice exhibit increased sensitivity to Escherichia coli infection but not to ischemia-reperfusion injury. Proc Natl Acad Sci U S A. 2006;103:1834-9. https://doi.org/10.1073/pnas.0510847103.

40. Vogel-Ciernia A, Wood MA. Examining object location and object recognition memory in mice. Curr Protoc Neurosci Editor Board Jacqueline N Crawley Al. 2014;69:8.31.1-8.31.17. https://doi.org/10.1002/0471142301.ns0831s69.

41. Wolf A, Bauer B, Abner EL, Ashkenazy-Frolinger T, Hartz AMS. A comprehensive behavioral test battery to assess learning and memory in 129S6/Tg2576 mice. PLoS One. 2016;11:e0147733. https://doi.org/10.1371/ journal.pone.0147733.

42. Walf AA, Frye CA. The use of the elevated plus maze as an assay of anxietyrelated behavior in rodents. Nat Protoc. 2007;2:322-8. https://doi.org/10. 1038/nprot.2007.44.

43. D'Hooge R, De Deyn PP. Applications of the Morris water maze in the study of learning and memory. Brain Res Brain Res Rev. 2001;36:60-90.

44. Van Dam D, Coen K, De Deyn PP. Cognitive evaluation of disease-modifying efficacy of donepezil in the APP23 mouse model for Alzheimer's disease. Psychopharmacology. 2008;197:37-43. https:/doi.org/10.1007/s00213-007-1010-x.

45. Van Dam D, Lenders G, De Deyn PP. Effect of Morris water maze diameter on visual-spatial learning in different mouse strains. Neurobiol Learn Mem. 2006;85:164-72. https://doi.org/10.1016/j.n/m.2005.09.006.

46. Chen Q, Tang J, Tan L, Guo J, Tao Y, Li L, et al. Intracerebral hematoma contributes to hydrocephalus after intraventricular hemorrhage via aggravating Iron accumulation. Stroke. 2015;46:2902-8. https://doi.org/10. 1161/STROKEAHA.115.009713.

47. Hovens IB, van Leeuwen BL, Nyakas C, Heineman E, van der Zee EA, Schoemaker RG. Postoperative cognitive dysfunction and microglial activation in associated brain regions in old rats. Neurobiol Learn Mem. 2015;118:74-9. https://doi.org/10.1016/j.nlm.2014.11.009.

48. Hovens IB, Nyakas C, Schoemaker RG. A novel method for evaluating microglial activation using ionized calcium-binding adaptor protein-1 staining: cell body to cell size ratio. Neuroimmunol Neuroinflammation. 2014;1:82-8.

49. Bao G-H, Ho C-T, Barasch J. The ligands of neutrophil gelatinase-associated lipocalin. RSC Adv. 2015;5:104363-74. https://doi.org/10.1039/C5RA18736B.

50. Bao G, Clifton M, Hoette TM, Mori K, Deng S-X, Qiu A, et al. Iron traffics in circulation bound to a siderocalin (Ngal)-catechol complex. Nat Chem Biol. 2010;6:602-9. https://doi.org/10.1038/nchembio.402.
51. Devireddy LR, Hart DO, Goetz DH, Green MR. A mammalian siderophore synthesized by an enzyme with a bacterial homolog involved in enterobactin production. Cell. 2010;141:1006-17. https://doi.org/10.1016/j. cell.2010.04.040

52. Devireddy LR, Gazin C, Zhu X, Green MR. A cell-surface receptor for lipocalin 24p3 selectively mediates apoptosis and iron uptake. Cell. 2005;123:1293305. https://doi.org/10.1016/j.cell.2005.10.027.

53. Xu G, Ahn J, Chang S, Eguchi M, Ogier A, Han S, et al. Lipocalin-2 induces cardiomyocyte apoptosis by increasing intracellular iron accumulation. J Biol Chem. 2012;287:4808-17. https://doi.org/10.1074/jbc.M111.275719.

54. Xiao X, Yeoh BS, Saha P, Olvera RA, Singh V, Vijay-Kumar M. Lipocalin 2 alleviates iron toxicity by facilitating hypoferremia of inflammation and limiting catalytic iron generation. Biometals Int J Role Met lons Biol Biochem Med. 2016;29:451-65. https://doi.org/10.1007/s10534-016-9925-5.

55. Srinivasan G, Aitken JD, Zhang B, Carvalho FA, Chassaing B, Shashidharamurthy $R$, et al. Lipocalin 2 deficiency dysregulates iron homeostasis and exacerbates endotoxin-induced sepsis. J Immunol. 2012; 189:1911-9. https://doi.org/10.4049/jimmunol.1200892.

56. Urrutia P, Aguirre P, Esparza A, Tapia V, Mena NP, Arredondo M, et al. Inflammation alters the expression of DMT1, FPN1 and hepcidin, and it causes iron accumulation in central nervous system cells. J Neurochem. 2013;126:541-9. https://doi.org/10.1111/jnc.12244.

57. McCarthy RC, Sosa JC, Gardeck AM, Baez AS, Lee C-H, Wessling-Resnick M. Inflammation-induced iron transport and metabolism by brain microglia. J Biol Chem. 2018;293:7853-63. https://doi.org/10.1074/jbc.RA118.001949.

58. Rathore Kl, Redensek A, David S. Iron homeostasis in astrocytes and microglia is differentially regulated by TNF- $a$ and TGF- $\beta 1$. Glia. 2012;60:73850. https://doi.org/10.1002/glia.22303.

59. Pelizzoni I, Zacchetti D, Campanella A, Grohovaz F, Codazzi F. Iron uptake in quiescent and inflammation-activated astrocytes: a potentially neuroprotective control of iron burden. Biochim Biophys Acta. 1832;2013: 1326-33. https://doi.org/10.1016/j.bbadis.2013.04.007.

60. You L-H, Yan C-Z, Zheng B-J, Ci Y-Z, Chang S-Y, Yu P, et al. Astrocyte hepcidin is a key factor in LPS-induced neuronal apoptosis. Cell Death Dis. 2017;8:e2676. https://doi.org/10.1038/cddis.2017.93.

61. Thomsen MS, Andersen MV, Christoffersen PR, Jensen MD, Lichota J, Moos T. Neurodegeneration with inflammation is accompanied by accumulation of iron and ferritin in microglia and neurons. Neurobiol Dis. 2015;81:108-18. https://doi.org/10.1016/j.nbd.2015.03.013.

62. Ferreira AC, Santos T, Sampaio-Marques B, Novais A, Mesquita SD, Ludovico $P$, et al. Lipocalin-2 regulates adult neurogenesis and contextual discriminative behaviours. Mol Psychiatry. 2018;23:1031-9. https://doi.org/10. 1038/mp.2017.95

63. Nairz M, Theurl I, Schroll A, Theurl M, Fritsche G, Lindner E, et al. Absence of functional Hfe protects mice from invasive Salmonella enterica serovar typhimurium infection via induction of lipocalin-2. Blood. 2009;1 14:3642-51. https://doi.org/10.1182/blood-2009-05-223354.

64. Nairz M, Schroll A, Haschka D, Dichtl S, Sonnweber T, Theurl I, et al. Lipocalin-2 ensures host defense against Salmonella typhimurium by controlling macrophage iron homeostasis and immune response. Eur J Immunol. 2015;45:3073-86. https://doi.org/10.1002/eji.201545569.

65. Bi F, Huang C, Tong J, Qiu G, Huang B, Wu Q, et al. Reactive astrocytes secrete Icn2 to promote neuron death. Proc Natl Acad Sci U S A. 2013;110: 4069-74. https://doi.org/10.1073/pnas.1218497110.

66. Egashira Y, Hua Y, Keep RF, Iwama T, Xi G. Lipocalin 2 and blood-brain barrier disruption in white matter after experimental subarachnoid hemorrhage. Acta Neurochir Suppl. 2016;121:131-4. https://doi.org/10.1007/ 978-3-319-18497-5_23.

67. Egashira Y, Hua Y, Keep RF, Xi G. Acute white matter injury after experimental subarachnoid hemorrhage: potential role of lipocalin 2. Stroke. 2014;45:2141-3. https://doi.org/10.1161/STROKEAHA.114.005307.

68. Shishido H, Toyota Y, Hua Y, Keep RF, Xi G. Role of lipocalin 2 in intraventricular haemoglobin-induced brain injury. Stroke Vasc Neurol. 2016; 1:37-43. https://doi.org/10.1136/svn-2016-000009.

69. Jin M, Kim J-H, Jang E, Lee YM, Soo Han H, Woo DK, et al. Lipocalin-2 deficiency attenuates neuroinflammation and brain injury after transient middle cerebral artery occlusion in mice. J Cereb Blood Flow Metab Off J Int Soc Cereb Blood Flow Metab. 2014;34:1306-14. https://doi.org/10.1038/jcbfm.2014.83.

70. Wirths $\mathrm{O}$, Bayer TA. Neuron loss in transgenic mouse models of Alzheimer's disease. Int J Alzheimers Dis. 2010;2010:723782. https://doi.org/10.4061/ 2010/723782 
71. Kjeldsen L, Cowland JB, Borregaard N. Human neutrophil gelatinaseassociated lipocalin and homologous proteins in rat and mouse. Biochim Biophys Acta. 2000;1482:272-83.

72. Ferreira AC, Pinto V, Mesquita SD, Novais A, Sousa JC, Correia-Neves M, et al. Lipocalin-2 is involved in emotional behaviors and cognitive function. Front Cell Neurosci. 2013;7:122. https://doi.org/10.3389/fncel.2013.00122.

73. Mucha M, Skrzypiec AE, Schiavon E, Attwood BK, Kucerova E, Pawlak R. Lipocalin-2 controls neuronal excitability and anxiety by regulating dendritic spine formation and maturation. Proc Natl Acad Sci U S A. 2011;108:1843641. https://doi.org/10.1073/pnas.1107936108.

Ready to submit your research? Choose BMC and benefit from:

- fast, convenient online submission

- thorough peer review by experienced researchers in your field

- rapid publication on acceptance

- support for research data, including large and complex data types

- gold Open Access which fosters wider collaboration and increased citations

- maximum visibility for your research: over $100 \mathrm{M}$ website views per year

At BMC, research is always in progress.

Learn more biomedcentral.com/submissions 